EPJ Web of Conferences 85, 02035 (2015)

DOI: $10.1051 /$ epjconf/20158502035

(C) Owned by the authors, published by EDP Sciences, 2015

\title{
Overview of HERMES Results on Exclusive Processes
}

\author{
Aram Movsisyan ${ }^{1, a}$ \\ on behalf of the HERMES Collaboration \\ ${ }^{1}$ INFN-University of Ferrara, Via Saragat 1, 44122 Ferrara, Italy
}

\begin{abstract}
An overview of HERMES results on measurements of hard exclusive electroproduction of real photons and mesons on hydrogen and deuterium targets is presented. Experimental measurements of hard exclusive processes are commonly described within the formalism of generalized parton distributions (GPDs), which provide a unified description of the structure of hadrons embedding longitudinal-momentum distributions (ordinary PDFs) and transverse-position information (form factors). The HERMES experiment at DESY Hamburg studies hard exclusive processes using polarized electron or positron beams from HERA and internal gas targets. Information about GPDs is gained from the measurements of asymmetries that appear in the azimuthal distributions of produced mesons and photons, together with studies of the azimuthal distribution of the decay products via spin-density matrix elements.
\end{abstract}

\section{Introduction}

Hard exclusive processes are discovered to provide a wealth of information about the bound state structure of hadrons in QCD as well as about the fundamental mechanisms that control hadron dynamics in high energy interactions. Currently the most comprehensive description of hard exclusive reactions is given within the framework of Generalized Parton Distributions (GPDs) [1-3]. The GPDs that are off-diagonal extensions of ordinary parton distributions provide a multidimensional description of the nucleon structure in terms of quark and gluon degrees of freedom. In contrast to form factors and parton distribution functions, which are one dimensional distributions, the GPDs contain a correlated information on transverse spatial and longitudinal momentum distributions of partons $[4,5]$, moreover the elastic form factors and parton distributions appear as moments and limiting cases of GPDs, respectively. Another interesting feature of GPDs is their sensitivity to the total angular momentum of partons in the nucleon according to Ji relation [3]. Generalized parton distributions depend upon four kinematic variables: the Mandelstam variable $t=\left(p-p^{\prime}\right)^{2}$, which is the squared momentum transfer to the nucleon with $p$ and $p^{\prime}$ representing the initial and final four-momentum of the nucleon; the average fraction $x$ of the nucleon's longitudinal momentum carried by the active parton under the consideration; half the difference of the fractions of the nucleon's longitudinal momentum carried by the active parton at the initial and final state, written as the skewness $\xi$; and $Q^{2}$, i.e., the negative square of the four-momentum of the virtual photon that mediates the lepton-nucleon scattering process.
Experimental information about GPDs is so far obtained from the measurements of hard exclusive leptonnucleon scattering processes. The hard exclusive processes on spin-1/2 nucleon are described by four leadingtwist, quark chirality conserving GPDs $(H, E, \widetilde{H}$, and $\widetilde{E})$, while certain experimental observables require a consideration of higher-twist contributions as well. In the case of spin-1 target, one needs nine GPDs to describe all experimental observables. The most commonly used experimental probe of GPDs is the Deeply Virtual Compton Scattering (DVCS) or hard leptoproduction of real photons $\ell+N \rightarrow \ell+N^{\prime}+\gamma$. During last decade DVCS process was extensively studied from both theoretical and experimental sides. Currently there exist several measurements by HERMES, CLAS, H1, ZEUS and Hall-A experiments. The main observables are the cross-sections, cross-section differences and azimuthal asymmetries. Apart from DVCS a complementary information on GPDs can be obtained from measurements of hard exclusive meson production processes $\ell+N \rightarrow \ell+N^{\prime}+V$. Unlike DVCS, which is well studied also from the theory side and even effects of next-to-leading order and next-to-leading twist are under control, the situation with hard meson production is in a less advanced stage. First of all the factorisation is proven not for all the amplitudes, thus the interpretation of the observations in terms of GPDs requires more sophisticated measurements with possible separation of different amplitudes. In addition also a good knowledge of meson distribution amplitudes is important. On the other side, measurements of exclusive meson production processes with different final state mesons $(\rho, \phi, \omega$ etc.) provide a unique opportunity to study the GPDs of various flavours.

\footnotetext{
ae-mail: aram.movsisyan @ desy.de
} 


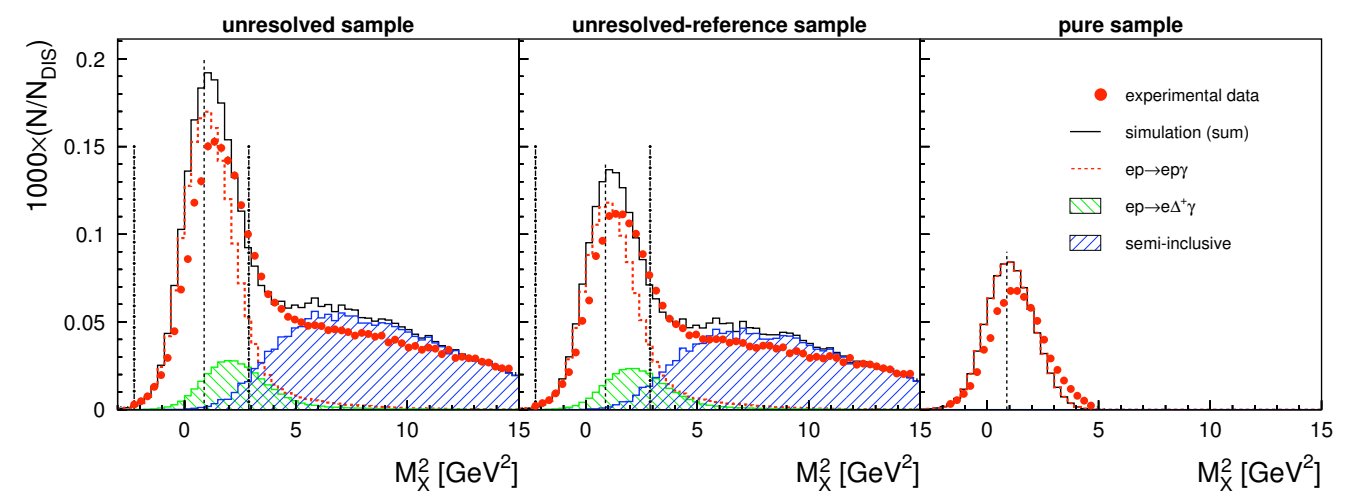

Figure 1. Missing mass squared distributions for three samples: unresolved (left), unresolved reference (middle) and pure sample (right).

\section{HERMES measurements of Deeply Virtual Compton Scattering}

Deeply Virtual Compton Scattering, or hard leptoproduction of real photons, where the photon is emitted by a struck quark in the nucleon, is currently considered to be the cleanest process that provides an access to GPDs. Beside DVCS, there is another process with the same initial and final state, Bethe-Heitler $(\mathrm{BH})$, where the final photon is radiated by an incoming or outgoing lepton. These processes are experimentally indistinguishable, and therefore the total cross section of hard leptoproduction of real photons contains an interference term $\mathcal{I}$, which depends on the charge of the lepton beam.

$$
\sigma \propto\left|\mathcal{T}_{B H}\right|^{2}+\left|\mathcal{T}_{D V C S}\right|^{2}+\mathcal{I}
$$

The individual terms from the cross section can be decomposed into Fourier harmonics in azimuthal angle $\phi$, which is defined as an angle between the lepton scattering plane and the photon production plane. Different Fourier components depend on different Compton Form Factors (CFF), which in turn are convolutions of hard scattering amplitudes with the corresponding GPDs [6].

At HERMES, the DVCS process is accessed through measurements of cross section asymmetries that appear in the azimuthal distributions of final state photons. Using data collected with longitudinally polarized electron and positron beams with both target polarization states, it is possible to measure asymmetries with respect to beam charge, beam polarization and target polarization alone and also with respect to their different combinations. As an example, the asymmetries in the cross section for scattering a longitudinally polarized electron/positron beam off a transversely polarized hydrogen target can be described as

$$
\begin{aligned}
d \sigma & =d \sigma_{U U}(\phi)\left\{1+e_{\ell} \mathcal{A}_{C}(\phi)+P_{\ell} \mathcal{A}_{L U}^{D V C S}(\phi)\right. \\
& +S_{\perp} \mathcal{A}_{U T}^{D V C S}\left(\phi, \phi_{S}\right)+e_{\ell} P_{\ell} \mathcal{A}_{L U}^{I}(\phi)+e_{\ell} S_{\perp} \mathcal{A}_{U T}^{I}\left(\phi, \phi_{S}\right) \\
& \left.+P_{\ell} S_{\perp} \mathcal{A}_{L T}^{D V C S}\left(\phi, \phi_{S}\right)+e_{\ell} P_{\ell} S_{\perp} \mathcal{A}_{L T}^{I}\left(\phi, \phi_{S}\right)\right\} .
\end{aligned}
$$

Here $d \sigma_{U U}$ is the cross section of scattering an unpolarized lepton beam off an unpolarized target averaged over both beam charges, $e_{\ell}$ and $P_{\ell}$ are the beam charge and polarisation respectively, and $S_{\perp}$ is the transverse target polarisation. The angle $\phi_{S}$ is defined as an angle between the lepton plane and the transverse component of the target polarisation. The asymmetries from Eq. 2 are farther decomposed into Fourier harmonics in azimuthal angles $\phi$ and $\phi_{S}$ and the corresponding asymmetry amplitudes are extracted simultaneously, using a maximum likelihood fit.

Two different analysis approaches were used at HERMES for the selection of BH and DVCS events. Since the data from 1996 up to 2005 were collected without the recoil detecter, it was not possible to measure complete final

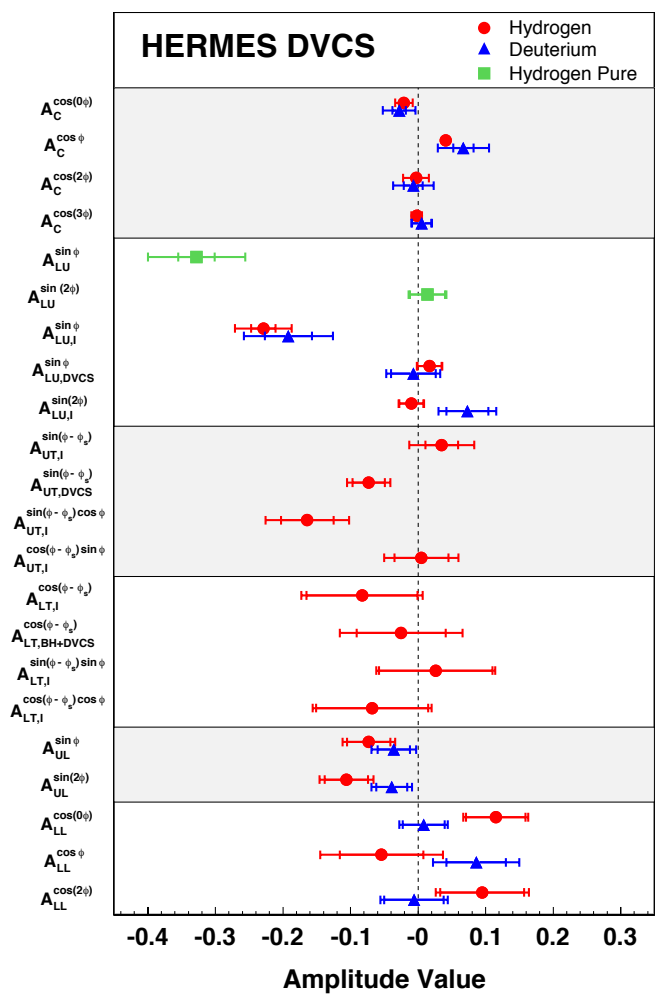

Figure 2. Overview of DVCS amplitudes integrated over the entire HERMES kinematic range. 


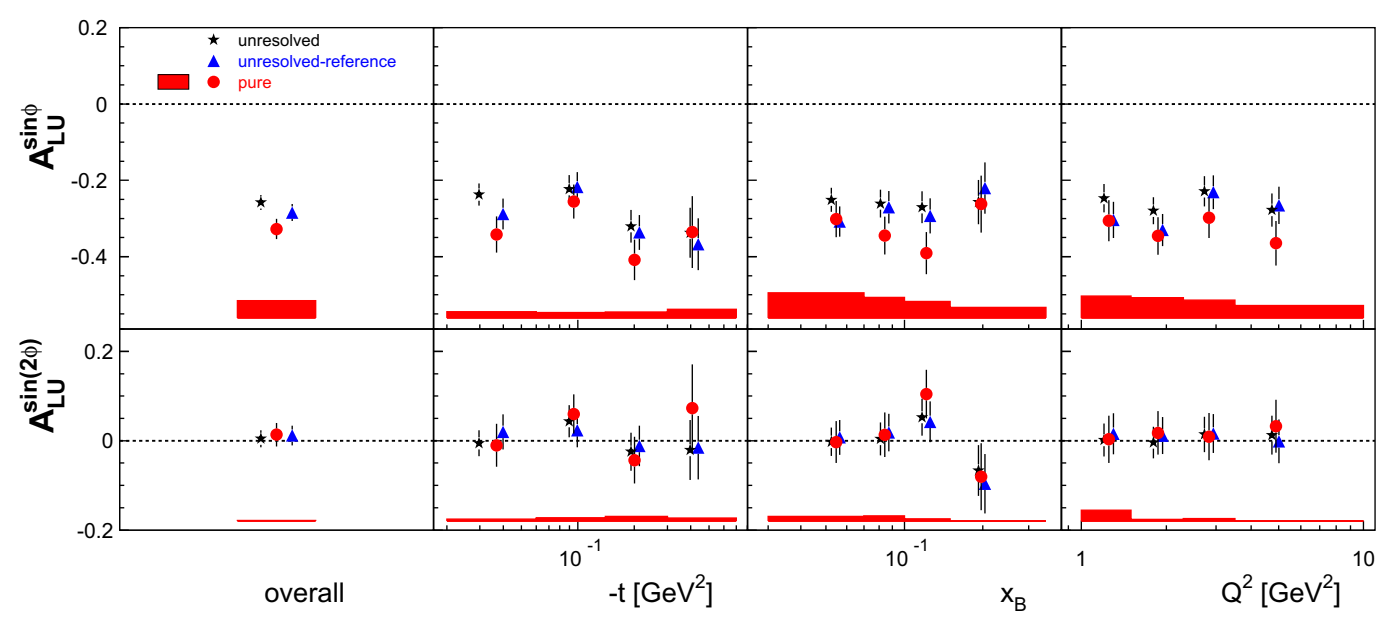

Figure 3. Results of single-charge beam-helicity asymmetry amplitudes for pure, unresolved and unresolved reference samples.

state of the process. Instead a missing mass technique was applied to achieve an exclusivity. A typical distribution of squared missing mass $M_{X}^{2}=\left(q+M_{p}-q^{\prime}\right)^{2}$ is shown in Fig. 1(left). Here $q^{\prime}$ is the four momentum of produced photon. The requirement $-2.25 \mathrm{GeV}^{2}<M_{X}^{2}<2.89 \mathrm{GeV}^{2}$ was applied to ensure the exclusivity of the event sample. As can be seen from Fig. 1, the selected exclusive sample receive's contribution (about $12 \%$ ) also from the associated processes, where the target proton excites to a resonant state and also from a semi-inclusive production of neutral pions (about $4 \%$ ). The measured asymmetry amplitudes were corrected for the contribution of semiinclusive pion production, while the contribution from associated processes could not be resolved and remained as a part of signal.

An overview of all extracted azimuthal asymmetry amplitudes corresponding to the entire HERMES kinematics is presented in Fig. 2 for both Hydrogen and Deuterium targets [7-9]. The amplitudes of the beam-helicity and beam-charge asymmetries $A_{L U}^{D V C S}(\phi), A_{L U}^{I}(\phi)$ and $A_{C}(\phi)$ are presented in the top panels of Fig. 2. A significant nonzero $\cos (\phi)$ and $\sin (\phi)$ amplitudes were observed respectively for beam-charge $A_{C}(\phi)$ and beam-helicity $A_{L U}^{I}(\phi)$ asymmetries. These two amplitudes are sensitive to the

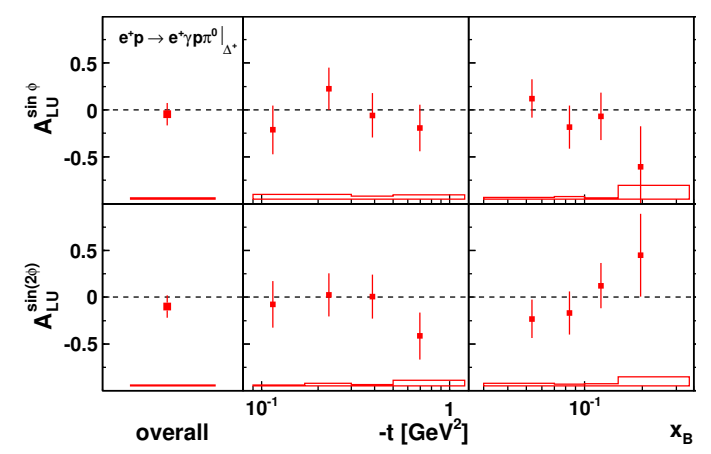

Figure 4. Amplitudes of single-charge beam-helicity asymmetry for the associated process $e p \rightarrow e \gamma p \pi^{0}$. real and imaginary parts of $\mathrm{CFF} \mathcal{H}$, respectively. The results for $\sin (\phi)$ amplitude of the charge-averaged beamhelicity asymmetry $A_{L U}^{D V C S}(\phi)$ are consistent with zero. In the bottom panels of Fig. 2, the results of the longitudinal single-target-spin $A_{U L}(\phi)$ and double-spin $A_{L L}(\phi)$ asymmetries are presented. For the case of hydrogen target, the leading amplitudes of these asymmetries are sensitive to the imaginary and real parts of CFF $\widetilde{\mathcal{H}}$, respectively. Also shown in Fig. 2 are the leading amplitudes of transverse single-target-spin and double-spin asymmetries, measured on Hydrogen target. The amplitude $A_{U T, I}^{\sin \left(\phi-\phi_{S}\right) \cos (\phi)}$, which has significant negative value, is sensitive to the imaginary part of CFF $\mathcal{E}$, while the amplitude $A_{L T, I}^{\sin \left(\phi-\phi_{S}\right) \sin (\phi)}$, which is consistent with zero, is sensitive to the real part of CFF $\mathcal{E}$.

During the last two years of HERA operation, the HERMES spectrometer was upgraded by a recoil detector [10] around the target cell. With the detection of recoiled proton it was possible to exclude the associated background and achieve a purity of BH/DVCS process of more then $99 \%$. The results of the single-charge beamhelicity asymmetry amplitudes $A_{L U}^{\sin (n \phi)}$ from the pure sample are also presented in Fig. 2 (green squares). In order to compare results under similar kinematic conditions, a

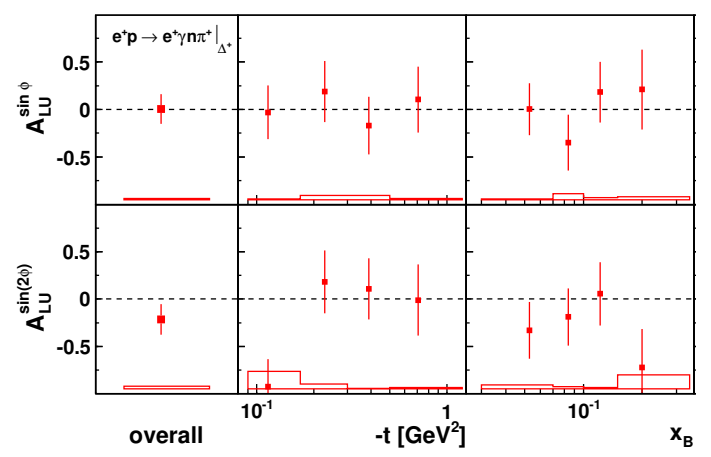

Figure 5. Amplitudes of single-charge beam-helicity asymmetry for the associated process $e p \rightarrow e \gamma n \pi^{+}$. 
"reference" event sample was created where in addition to the selection criteria used for the unresolved sample a "hypothetical" proton with 3-momenta calculated from the 3-momenta of electron and photon was required to be in the recoil detector acceptance. For this subsample, background conditions are very similar to those for the unresolved sample as can be seen from comparison of missing mass distributions on Fig. 1. Hence, it can be used for comparison with the background-free measurement. The results of single-charge beam-helicity asymmetry amplitudes for unresolved, unresolved reference and pure samples are compared in Fig. 3. Here, for the selection of pure event sample a kinematic event fitting technique was used, that allowed to achieve a purity above $99 \%$. With the measured kinematics of recoiled particle it was also possible to study the associated processes separately. Particularly two channels were investigated at HERMES $e p \rightarrow e \gamma p \pi^{0}$ and $e p \rightarrow e \gamma n \pi^{+}$in the $\Delta$ resonance region [11]. The results of single-charge beam-helicity asymmetry amplitudes of these channels are shown in Figs. 4 and 5 respectively. The obtained results of the amplitudes are consistent with zero for both channels, suggesting that the asymmetries of associated DVCS process can be treated as a dilution also for the other DVCS measurements at HERMES. Moreover they can be used to constrain models of transition GPDs.

\section{HERMES results on hard exclusive production of mesons}

In a single photon exchange approximation the theoretical description of the hard exclusive meson production processes can be given in two alternative approaches. Throughout many years the features of the process were successfully described in terms of Vector Meson Dominance model [12]. Hear the virtual photon, emitted by an incoming lepton, fluctuates into a vector meson, and the interaction of the later one with the nucleon is described using Regge phenomenology. The second approach is based on perturbative QCD description of the process, in terms of handbag diagrams [13]. Here the GPDs are involved in the parameterisation of the non-perturbative part of the diagrams. In pQCD approach the virtual photon dissociates into a $q \bar{q}$ pair, whose interaction with the nucleon results in a formation of final state meson. The interaction can proceed via two distinct mechanisms: quark-antiquark or two-gluon exchange, therefore providing an information also about gluon GPDs. In the Regge picture the interaction mechanism with two-gluon exchange is similar to the exchange of the pomeron, while the interaction mechanism with quark exchange is similar to the exchange of secondary reggeons, with either natural $\left(\omega, \rho, a_{2}\right)$ or unnatural $\left(\pi, a_{1}\right)$ parity. In context of GPDs, the natural parity exchange processes are described by GPDs $H$ and $E$, while in the description of unnatural parity exchange processes the GPDs $\widetilde{H}$ and $\widetilde{E}$ are involved. As a consequence, for the proper description of the experimental observables in terms of GPDs, it is of particular importance to distinguish relative contributions of various production mecha-

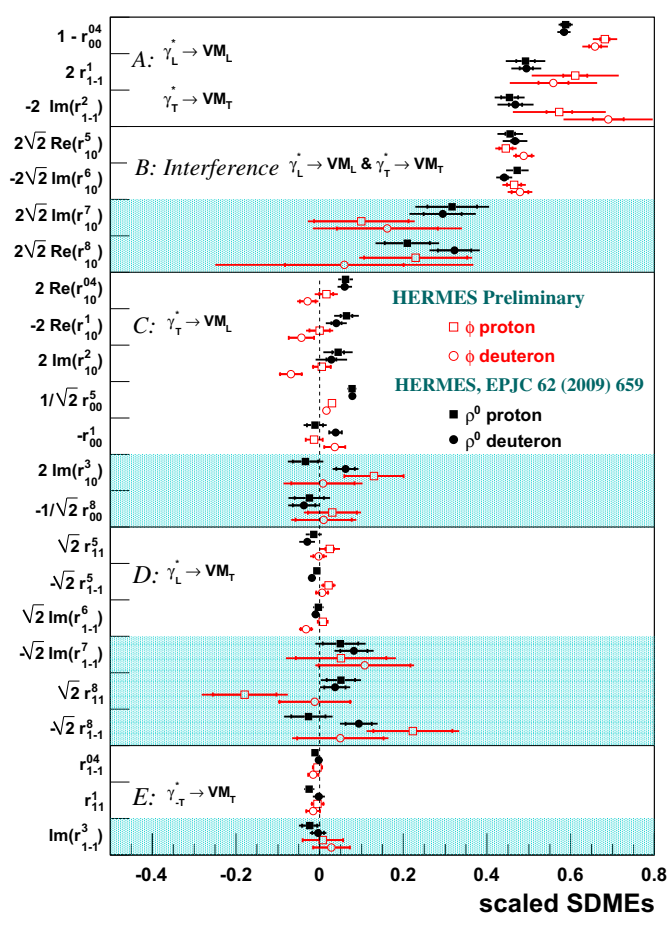

Figure 6. SDMEs of $\phi$ and $\rho^{0}$ mesons.

nisms in the given kinematic conditions of the particular measurement.

At HERMES the hard exclusive production of vector mesons was studied through measurements of Spin Density Matrix Elements (SDMEs) [14], which in turn are related to different helicity amplitudes. SDMEs are commonly used for the description of spin transfer from the virtual photon $\gamma^{*}$ to the vector meson. The scattering of unpolarized leptons off an unpolarized targets is described by 15 SDMEs, while for the scattering of longitudinally polarized leptons off an unpolarized targets, 8 additional SDMEs are required for the description of the process. The SDMEs can be obtained from decay angular distributions in the vector meson production.

The measurements of hard exclusive vector meson production, presented below were done on both Hydrogen and Deuterium targets and the results of SDMEs were extracted for $\rho, \phi$ and $\omega$ mesons in the following kinematic range: $1 G e V^{2}<Q^{2}<7 G e V^{2},-t<0.4 G e V^{2}$ and $2 \mathrm{GeV}<W<6.3 \mathrm{GeV}$. The extraction of SDMEs was performed using unbinned maximum likelihood fitting technique to fit the azimuthal distributions of decay products. The details of the exclusive event selection, SDME extraction, and the propagation of systematic uncertainties are presented in [15].

Results of 23 SDMEs for exclusive $\rho^{0}$ and $\phi$ meson production are presented in Fig. 6 for both Hydrogen and Deuterium targets corresponding to the entire HERMES kinematic range. The SDMEs are grouped into different classes corresponding to different spin transitions between virtual photon and vector meson. The SDMEs measured with unpolarized (polarized) beam are displayed in the unshaded (shaded) areas. The measured SDMEs of class A, 


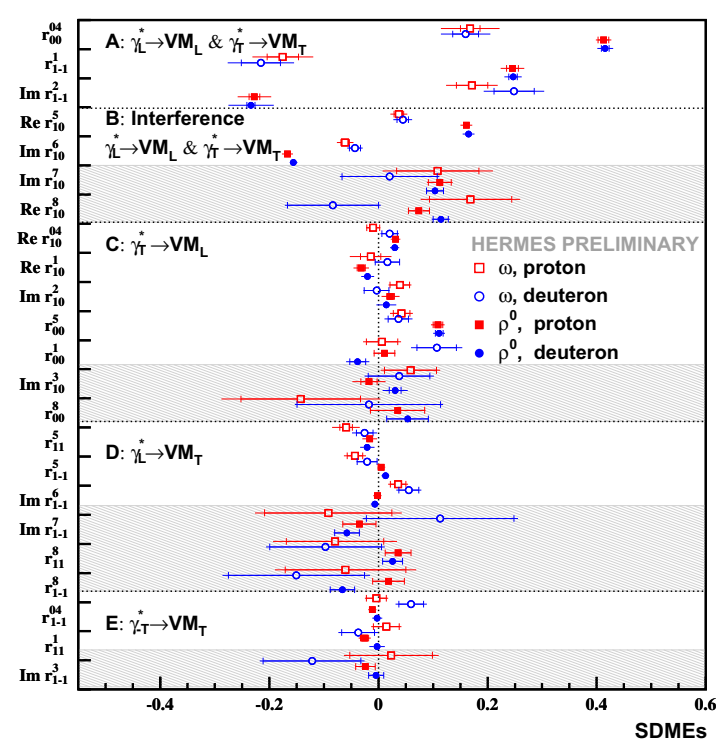

Figure 7. SDMEs of $\omega$ and $\rho^{0}$ mesons.

that correspond to the diagonal $\left(\gamma_{L}^{*} \rightarrow V_{L}\right.$ and $\gamma_{T}^{*} \rightarrow V_{T}$ ) transitions, and class $\mathrm{B}$, that correspond to the interference of the diagonal transition amplitudes, have significant non-zero values for both $\rho^{0}$ and $\phi$ mesons. There is in average $10-20 \%$ difference between results for $\rho^{0}$ and $\phi$ meson productions. Significant non-zero values of SDMEs of class $C$ are observed for $\rho^{0}$ meson. One of the SDMEs differs from zero by more than ten standard deviations of the total experimental uncertainty. This indicates the presence of a production mechanism that does not conserve s-channel helicity, and the non diagonal transition amplitudes are not negligible for the $\rho$ meson production. The $\phi$ meson SDMEs of the same class are consistent with zero. The SDMEs of classes D and E are also consistent with zero for both mesons except from the polarized $\rho^{0}$ SDMEs, which show slightly positive values. No significant differences are observed between SDMEs measured on Hydrogen and Deuterium targets.

Fig. 7 shows the results of 23 SDMEs for exclusive $\omega$ meson production on both Hydrogen and Deuterium targets. The results are shown in comparison with the SDMEs for $\rho$ meson. As in the case of $\rho$ and $\phi$ mesons, also for the $\omega$ meson the SDMEs of classes A and B significantly differ from zero. SDMEs of classes $\mathrm{C}$ and $\mathrm{E}$ are also consistent with zero, while SDMEs of class D show small deviation from zero, suggesting a violation from schannel helicity conservation and a presence of non negligible contribution from non diagonal amplitudes. Within the total experimental uncertainty the SDMEs of $\omega$ meson measured on both targets are consistent. The comparison between SDMEs for $\omega$ and $\rho$ mesons shows significant differences. This can be explained by the significant contribution from the unnatural parity exchange mechanism in the production of $\omega$ mesons. Relative contribution of natural and unnatural parity exchange mechanisms can be studied from the measured SDMEs considering the following combinations: $U_{1}=1-r_{00}^{04}+2 r_{1-1}^{04}-2 r_{11}^{1}-2 r_{1-1}^{1}$, $U_{2}=r_{11}^{5}+r_{1-1}^{5}$ and $U_{3}=r_{11}^{8}+r^{1-1}$. Under the assumption that the natural parity exchange mechanism has the dominant contribution, the above given quantities are expected to vanish. Current measurements show that the quantity $U_{1}$ for $\rho$ meson has a value of 0.125 and differs from zero by more than two standard deviations of the total experimental uncertainty, suggesting a non negligible contribution from unnatural parity exchange mechanism. The same quantity for the $\omega$ meson has also a significant positive value, moreover it is larger than unity, which can be explained by the dominance of unnatural parity exchange amplitudes over the natural parity exchange ones.

From the measured results of SDMEs it is possible to study also the longitudinal-to-transverse cross-section ratio and also phase differences between various amplitudes. These results for the $\rho$ meson production are available in reference [15], and for the $\omega$ and $\phi$ mesons will be available in the upcoming publications.

Fig. 8 shows measured results of transverse target-spin asymmetry amplitudes in exclusive $\pi^{+}$production as a function of $-t, x_{B}$ and $Q^{2}$ [16]. Of special interest is the amplitude $A_{U T}^{\sin \left(\phi-\phi_{S}\right)}$, which is sensitive to GPDs $\widetilde{H}$ and $\widetilde{E}$. This amplitude was found to be consistent with zero, suggesting a possible dominance of GPD $\widetilde{E}$ over $\widetilde{H}$. The rest of the amplitudes are compatible with zero except $A_{U T}^{\sin \left(\phi_{S}\right)}$

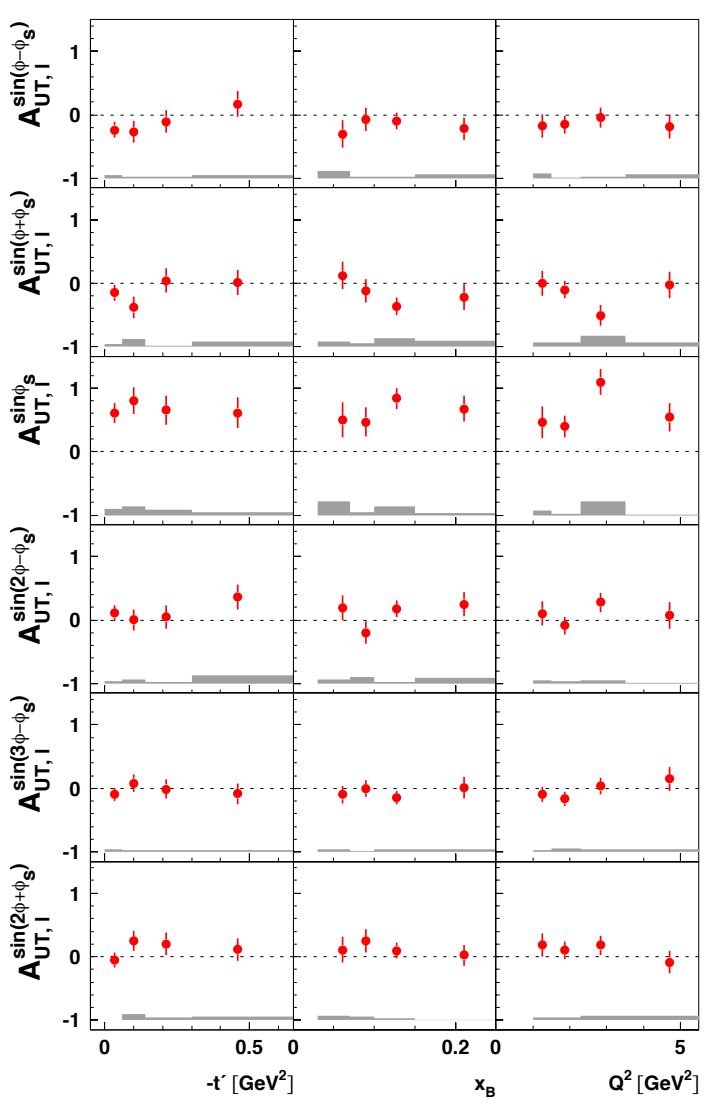

Figure 8. Amplitudes of transverse target-spin asymmetry in exclusive $\pi^{+}$production. 
indicating a significant contribution from the transverseto-longitudinal helicity transition of the virtual photon.

The presented experimental results of hard exclusive processes can provide strong constrains on various GPD models and can be used for fitting various free parameters of the particular GPD model. HERMES results have been already used with several groups of theoreticians. An examples of such a comparison of HERMES results with a specific GPD model and also there usage in the global fitting procedure of world data can be found in references [17-20].

\section{References}

[1] D. Muller, et al., Fortsch. Phys. 42101 (1994)

[2] A. V. Radyushkin, Phys. Lett. B380 417 (1996), Phys. Rev. D56 5524 (1997)

[3] X. Ji, Phys. Rev. Lett. 78610 (1997), Phys. Rev. D55 7114 (1997)

[4] J. P. Ralston, B. Pire, Phys. Rev. D66 111501 (2002)

[5] M. Burkardt, Phys. Rev. D62 071503 (2000); Erratumibid C31 277 (2003)

[6] A.V. Belitsky, D. Muller, A. Kirchner, Nucl. Phys. B629 323 (2002).
[7] A. Airapetian et al.,[HERMES Collab.], JHEP 07032 (2012), JHEP 06019 (2010), JHEP 06066 (2008), Phys. Lett. B 70415 (2011).

[8] A. Airapetian et al.,[HERMES Collab.], Nucl. Phys. B829 1 (2010), Nucl. Phys. B842 265 (2011).

[9] A. Airapetian et al.,[HERMES Collab.], JHEP 10042 (2012).

[10] A. Airapetian et al.,[HERMES Collab.], JINST 8 P05012 (2013).

[11] A. Airapetian et al.,[HERMES Collab.], JHEP 01 077 (2014).

[12] J. J. Sakurai, Ann. Phys. 111 (1960)

[13] S. J. Brodsky et al., Phys. Rev. D50 3134 (1994)

[14] K. Schillimng, G. Wolf, Nucl. Phys. B61 381 (1973)

[15] A. Airapetian et al., [HERMES Collab.], EPJ C62 659 (2009)

[16] A. Airapetian et al.,[HERMES Collab.], Phys. Lett. B682 345 (2010).

[17] K. Kumericki, et al., Nucl. Phys. B841 1 (2010), Phys. Part. Nucl. 45 4, 723 (2014.)

[18] M. Guidal, Phys. Lett. B693 17 (2010),

[19] M. Guidal, et al., Rept. Prog. Phys. 76066202 (2013),

[20] S. V. Goloskokov, P. Kroll, EPJ A50 9, 146 (2014), EPJ C74 725 (2014), EPJ C53 367 (2008). 